International Journal of Modern Physics B

Vol. 31, No. 12 (2017) 1750181 (18 pages)

(C) The Author(s)

DOI: $10.1142 / \mathrm{S} 0217979217501818$

\title{
The influence of passenger flow on the topology characteristics of urban rail transit networks
}

\author{
Yingyue Hu, Feng Chen*, Peiwen Chen and Yurong Tan \\ School of Civil Engineering, Beijing Jiaotong University, \\ Beijing 100044, P. R. China \\ *fengchen@bjtu.edu.cn
}

Received 15 December 2016
Revised 17 February 2017
Accepted 7 April 2017
Published 20 April 2017

Current researches on the network characteristics of metro networks are generally carried out on topology networks without passenger flows running on it, thus more complex features of the networks with ridership loaded on it cannot be captured. In this study, we incorporated the load of metro networks, passenger volume, into the exploration of network features. Thus, the network can be examined in the context of operation, which is the ultimate purpose of the existence of a metro network. To this end, section load was selected as an edge weight to demonstrate the influence of ridership on the network, and a weighted calculation method for complex network indicators and robustness were proposed to capture the unique behaviors of a metro network with passengers flowing in it. The proposed method was applied on Beijing Subway. Firstly, the passenger volume in terms of daily origin and destination matrix was extracted from exhausted transit smart card data. Using the established approach and the matrix as weighting, common indicators of complex network including clustering coefficient, betweenness and degree were calculated, and network robustness were evaluated under potential attacks. The results were further compared to that of unweighted networks, and it suggests indicators of the network with consideration of passenger volumes differ from that without ridership to some extent, and networks tend to be more vulnerable than that without load on it. The significance sequence for the stations can be changed. By introducing passenger flow weighting, actual operation status of the network can be reflected more accurately. It is beneficial to determine the crucial stations and make precautionary measures for the entire network's operation security.

Keywords: Urban rail transit; complex networks; weighting network; attack; robustness.

PACS numbers: 89.75.Fb, 89.40.-a, 05.90.+m

This is an Open Access article published by World Scientific Publishing Company. It is distributed under the terms of the Creative Commons Attribution 4.0 (CC-BY) License. Further distribution of this work is permitted, provided the original work is properly cited.

*Corresponding author. 


\section{Introduction}

Transport infrastructure is one of the fundamental factors in a nation's development. In a metropolis like Beijing, urban rail transit is the preferred travel mode of citizens because of its speed, convenience, high efficiency, punctuality and environmental protection. However, owing to its very large passenger volume, urban rail transit can be a primary target for terror attacks, such as in the cases of explosions on the London Underground and Madrid railways. Meanwhile, with the rapid increase in passenger flow volume and the scale of metro networks, operational accidents can cause trains in local section stops or can even lead to severe casualties and breakdown of the entire network system. Determining the primary target stations for terror attacks and analyzing the capacity of the network system after an attack has become a research emphasis in anti-terrorism measures and networked operation safety in urban rail transit.

Currently, research regarding networked transport systems focuses mainly on the basic theory and methods of complex networks..$^{1-6}$ Some research ${ }^{7-11}$ suggests the degree to which a transport network obeys a power law distribution or exponential distribution. Metro networks also have the characteristics of small-world networks and scale-free networks, $\frac{12}{2}$ which obey an exponential distribution, $\frac{13}{,}$ Poisson degree distribution $\frac{14}{}$ and power law distribution. $\frac{15}{}$ These are all involved in the analysis of topological structure in unweighted networks. Some research based on weighed networks for social cooperation ${ }^{16,17}$ suggests that the node strength and degree distribution of weighted networks appear to obey the fat-tailed power law distribution. Research on transport networks usually studies airline networks considering the number of flights as a weight. In this area of research, it has been discovered that the clustering coefficient of a weighted network is different from that of an unweighted network. ${ }^{7,18}$ Bagler $^{8}$ analyzed the distribution characteristics of the eigenvalues of weighted complex networks in airline networks. Airline networks are a type of space network: any two points within a feasible distance can be linked by a single-trip airline and little interference exists among different airlines, which is quite different from metro networks. Metro networks belong to the group of networks in L space: a trip passes many stations, and the passenger flow volume is limited by the system's capacity. Previous studies on the reliability of transport networks have also focused on unweighted networks, ${ }^{19-21}$ and few considered passenger flow as a weight for evaluating the reliability of weighted networks. Nagurney ${ }^{22}$ once considered the average passenger flow in each origin and destination pair in unit time as the evaluation index of network reliability and analyzed the network reliability by simulating the scenario where one node or side is attacked at one time. However, network reliability in the condition of continuous attack was not considered.

In summary, existing literatures on subject of the characteristic and network robustness of urban rail transit mostly only focused on the analysis of the network structure, ignoring the essential function of transportation network. The study of 
networks introducing passenger flow as a weight is rare. Furthermore, there is a lack of systematic comparison of complex network characteristic values between weighted and unweighted networks in the field of urban rail transit operation research. Analysis on the robustness of weighted networks for urban rail transit under a continuous attack is also scarce. The necessity and feasibility of taking the factor of passenger flow into consideration when studying networked urban rail transit is shown as follows. Firstly, the aim of establishing the rail transportation network is to meet the need of accomplishing the passenger transportation with higher speed and larger quantity. It is far away from the reality to dig the topology characteristic of the rail transportation network without considering the influence of passenger. Thus, it is the only way by supplementing the passenger information to the edge and node of the rail transportation network to simulate the network characteristic in real world more accurately. Secondly, the characteristics of passenger flow in different edge or node will vary with different passenger volume. There is strong relevance between the formation of urban rail transit's scale and morphology and passengers demand and flow rules. The relevance of stations is enhanced by passenger flow volume between them, elevating the importance of certain sections and stations. Thirdly, passengers have subjective initiative; thus, they can choose the optimal route based on the shortest route and degree of congestion. Finally, with the wide use of automatic fare collection (AFC) systems in urban rail transit and correspondingly massive amount of data regarding passenger flow, effectively using this data resource to improve service quality in urban rail transit is an emerging research emphasis.

In order to reveal better, the significance of considering essential function of transportation network when researching the urban rail transit network characteristics, the factor of passenger flow was introduced and the corresponding calculation method on network characteristic value was proposed. Then the successive network failure scenario assumption was set and the robustness of the network under attack was analyzed through the variation of the characteristic value. Finally, the results of the network robustness were compared with the results without considering passenger flow, such verifying is the necessity of considering the passenger flow when studying network characteristics. New characteristics can thus be revealed and city planners will be provided with not only a better understanding regarding the laws of urban rail transit networks but also the theoretical support for methods of effective management and operational safety.

\section{Materials and Methods}

The physical network of urban rail transit is composed of stations and lines. Figure 1 shows the network of the Beijing Subway. Figure 2 shows the accumulated section passenger flow volume in Beijing Subway network in a certain day. The width of the line indicates the quantity of the passenger flow volumes. Clearly, significant differences exist in passenger flow volumes for different stations and lines, as shown 


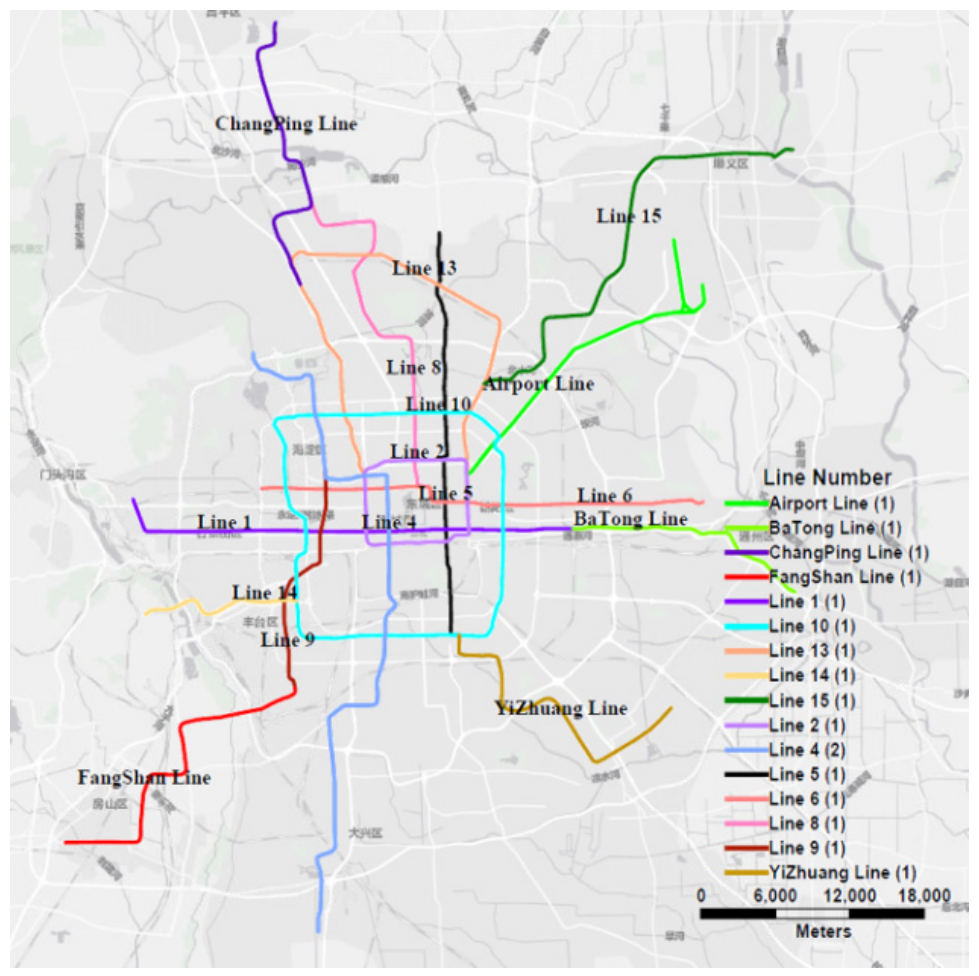

Fig. 1. (Color online) The Beijing Subway network in 2014.

in Fig. 2. The planning of lines serves passengers' travel demands. Meanwhile, travel demand also promotes the further development of the network. In this study, unweighted and weighted networks, respectively, describe models of the physical network by itself and with consideration of the travel characteristics of passenger flow.

\section{A. Unweighted networks}

An undirected graph is defined as $G=\{V, U\}$, where $\{V\}$ is the vertex of graph $\mathrm{G}$, which denotes the stations in an urban rail transit network. $\{U\}$ is the side of graph $\mathrm{G}$, which denotes the sections between each station. The urban rail transit network can be simplified as an unweighted network with vertices and sides.

\section{B. Weighted networks}

We introduce the passenger flow in each section as a set of weights $\{W\}$. Neighboring stations can be denoted as nodes $i$ and $j$, and the segment between them is $u_{i j}$. $w_{i j}$ is the weight, meaning the bidirectional passenger flow volume for a section per unit time. The unit time can be set based on the need; it can be either one hour or one day. 


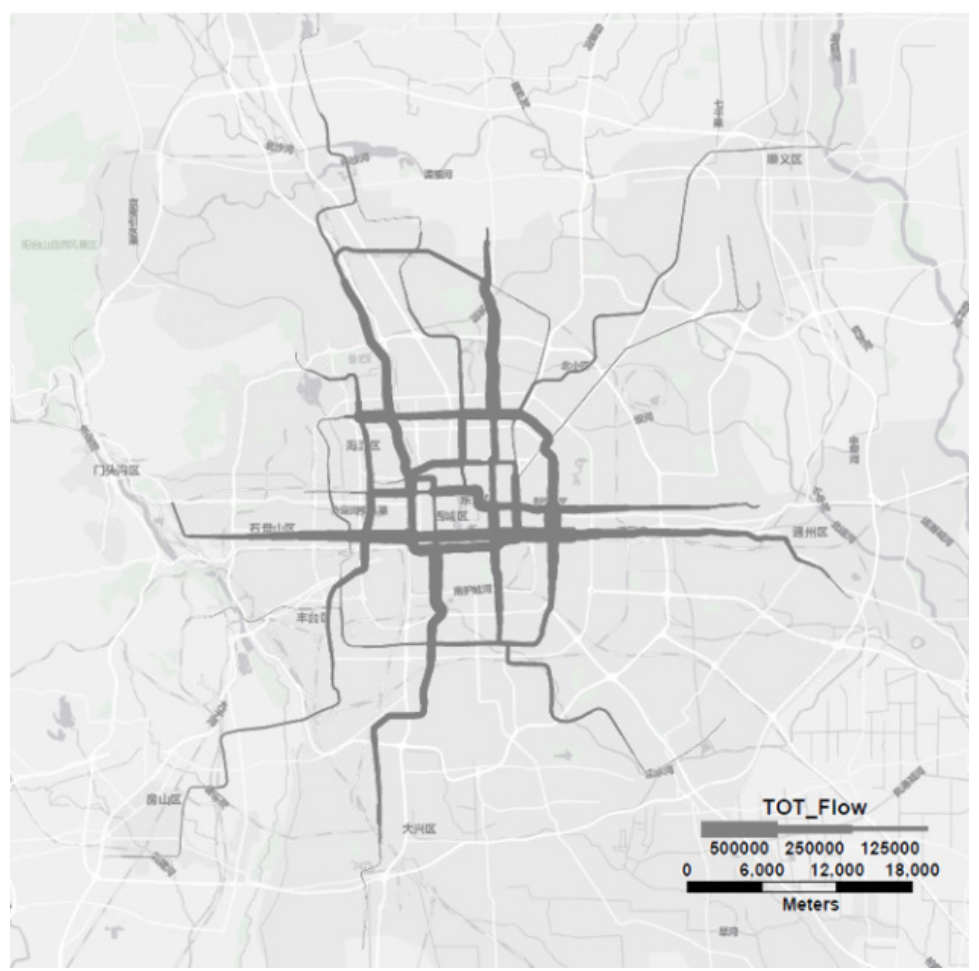

Fig. 2. The passenger flow distribution in Beijing Subway over one day in 2014 .

\subsection{Topological analysis of unweighted and weighted networks}

The parameters of complex unweighted and weighted networks are set in this section.

\section{A. Degree and node strength}

In an unweighted network, the degree of a node is defined as the number of connections the node has with other nodes, as shown in Eq. (1). In a weighted network, a node's strength is defined as the sum of its connecting sides' weights, as shown in Eq. (2).

$$
\begin{aligned}
k_{i} & =\sum_{j=1}^{n} a_{i j}, \\
S_{i} & =\sum_{j \in V} a_{i j} \cdot w_{i j},
\end{aligned}
$$

where $a_{i j}$ are elements of the adjacency matrix and $w_{i j}$ is the side weight of nodes $i$ and $j$. 
B. Clustering coefficient

In unweighted networks, the clustering coefficient $C$ denotes the ratio of $u_{i}$, the number of actual existing sides between station $v_{i}$ and its neighboring station $n_{i}$, to the number of possible sides between $v_{i}$ and $n_{i}$, as in Eq. (3). The clustering coefficient is combined with the side weight from a weighted network as shown in Eq. (4).

$$
\begin{aligned}
C_{i} & =\frac{u_{i}}{n_{i}\left(n_{i}-1\right) / 2} \\
C_{i}^{w} & =\frac{1}{s_{i}\left(k_{i}-1\right)} \sum_{j, k} \frac{w_{i j}+w_{i k}}{2} a_{i j} a_{i k} a_{j k}
\end{aligned}
$$

where $k_{i}$ is the degree of node $i$ and $a_{i j}$ are elements of the adjacency matrix.

The clustering coefficient $C$ of the unweighted network (or $C^{w}$ of the weighted network) is the average of all the nodes' clustering coefficients, as given in Eq. (5).

$$
C\left(\text { or } C^{w}\right)=\frac{1}{N} \sum_{i}^{n} C_{i}\left(\text { or } C_{i}^{w}\right),
$$

where $N$ is the number of stations in the network.

C. Shortest paths analysis

In unweighted networks, the length of the shortest path $d_{i j}$, denotes the number of sections in the shortest path between station $i$ and station $j$, whereas in weighted networks, the shortest path denotes the path in which the passenger flow volume of sections is smallest. We define the normalized passenger flow volume of section $d_{i j, s}^{w}$ as the length of the shortest path, given by Eq. (6). The average path length $L$ of the unweighted network (or the average path length $L^{w}$ of the weighted network) is calculated as in Eq. (7).

$$
\begin{aligned}
d_{i j, s}^{w} & =\frac{d_{i j}^{w}}{\left(\sum_{j \in v(i)} w_{i j}\right) / U}=\frac{\min _{1 \leq l \leq m}\left\{\sum_{x=1}^{n} w_{l}^{x}, x=1,2, \ldots, n\right\}}{\left(\sum_{j \in v(i)} w_{i j}\right) / U}, \\
L\left(\text { or } L^{w}\right) & =\frac{2}{N(N-1)} \sum_{i \geq j} d_{i j}\left(\text { or } d_{i j, s}^{w}\right),
\end{aligned}
$$

where $m$ is the number of shortest paths between stations $i$ and $j, w_{l}^{x}$ is the weight of the side $x$ of the path $l$ between nodes $i$ and $j, n$ is the number of sides of path $m, U$ is the total number of sides in the network, and $N$ means the total number of nodes in the network.

\section{Betweenness}

In an urban rail transit network, the shortest path is an important means of route selection. In an unweighted network, the betweenness $B C_{i}$ is defined as the ratio of the number of shortest paths passing station $i$ to the total number of shortest paths in the network, as in Eq. (8). Based on the definition of the loading intensity of passenger flow, in a weighted network, betweenness $B C_{i}^{w}$ is the ratio of the sum 
of weights in the shortest paths passing station $i$ to the sum of weights in all the shortest paths in the network, as in Eq. (9).

$$
\begin{aligned}
B C_{i} & =\frac{\sum_{s \neq t} n_{s t}^{i}}{\sum_{s \neq t} n_{s t}}, \quad(s, t \in V), \\
B C_{i}^{w} & =\frac{\sum_{l=1}^{n^{i}} \sum_{x \in l} w_{l}^{i, x}}{\sum_{l=1}^{n} \sum_{x \in l} w_{l}^{x}},
\end{aligned}
$$

where $n_{s t}$ is the number of shortest paths in the network, $n_{s t}^{i}$ is the number of shortest paths passing node $i$, between nodes $s$ and $t, w_{l}^{i, x}$ is the weight of side $x$ of path $l$ passing station $i, n$ is the number of weighted shortest paths in the network, and $n^{i}$ is the number of shortest paths passing node $i$.

\subsection{Analysis of network robustness}

Network robustness is the capacity of the network to maintain its operation after suffering an emergency or attack. When an urban rail transit network station is attacked, the passenger flow volume in the network is reallocated. In order to reasonably analyze the influence of weights on the evaluation of network robustness and further study its relevance, assumptions are made for a network attack as follows:

(1) A station suffering an attack means that the sides connecting the station are all damaged. Network attack is presumed to be continuous; that is, nodes are deleted from the network one by one.

(2) There are three attack strategies analyzed in this study: stochastic attacks, largest degree or largest strength node-based attacks and highest betweenness node-based attacks. The attack strategies on the urban transit network in real world can be sorted into two types. One is the stochastic attack, which can be regarded as several stations are subject to random successive failure. The other is the deliberate attack such as terrorist attacks. Usually the station with larger node degree (or node strength) or node betweenness gains more significance in the network. Thus, the stations are ranked according to the node degree (or node strength) and node betweenness from larger to smaller separately. The deliberate attack strategies obeying these two sequence are called largest degree or largest-strength node-based attacks and highest betweenness nodebased attacks separately.

(3) Apart from selecting average path length as the index for evaluation of network robustness, global efficiency is introduced as a reference. Its definition is shown as follows:

The efficiency between two nodes $\varepsilon_{i j}$ is the reciprocal of the shortest path length between the nodes. The global efficiency of the unweighted network $E_{\text {glob }}$ (or the global efficiency of the weighted network $\left.E_{\text {glob }}^{w}\right)$ is the average of the efficiencies of 
each pair of nodes, given in Eq. (10).

$$
E_{\text {glob }}\left(\text { or } E_{\text {glob }}^{w}\right)=\frac{1}{N(N-1)} \sum_{i \neq j} \varepsilon_{i j}=\frac{1}{N(N-1)} \sum_{i \neq j} \frac{1}{d_{i j}\left(\text { or } d_{i j}^{w}\right)},
$$

where $N$ is the total number of stations and $d_{i j}$ is the shortest path length between the pair of stations $i$ and $j\left(d_{i j}^{w}\right.$ is used to calculate the global efficiency of the weighted network).

\section{Results and Discussion}

This section presents the results of application of the proposed approach for the Beijing Subway. Until 2014, the Beijing Subway network had 18 lines, 277 stations and $527 \mathrm{~km}$ of track in operation. Its single-day ridership was close to 500 million. Beijing Subway is one of the most complicated transport network systems in the world and has an enormous passenger flow volume, making it an ideal case for convincing analysis.

\subsection{Comparison between parameters of topology structure and weighted network structure}

The data used in this study are origin-destination (OD) data from the AFC system in Beijing Subway taken on September 11th of 2014. The data includes passenger IDs and the number of departure and destination stations. MATLAB is used for programming to find the path between every ID's origin and destination nodes in the network and to define the shortest paths for specific passengers. Then the passenger flow in the network can be allocated based on an 'all or none' assignment model, thereby calculating the passenger flow volume for every section, which is considered as the weight for the corresponding side. Next, the parameters of complex unweighted and weighted networks can be calculated and compared.

\subsubsection{Clustering coefficient}

In the topology structure of the Beijing Subway network, only three stations Qilizhuang, Liuliqiao and Xiju — form a triangle. Thus most station nodes' clustering coefficients are 0 , which means that the clustering characteristic of this subway network is feeble and that the network's internal relationships are relatively simple. In the weighted network, clustering coefficients are also close to 0.

\subsubsection{Distribution of degree and node strength}

In the unweighted network, there are 16 stations with a degree of one, 179 stations with a degree of two, five stations with a degree of three, 26 stations with a degree of four, and one station (Xizhimen) with a degree of five, as shown in Fig. 3. The average degree of the entire network is 2.19 . In the weighted network, the 


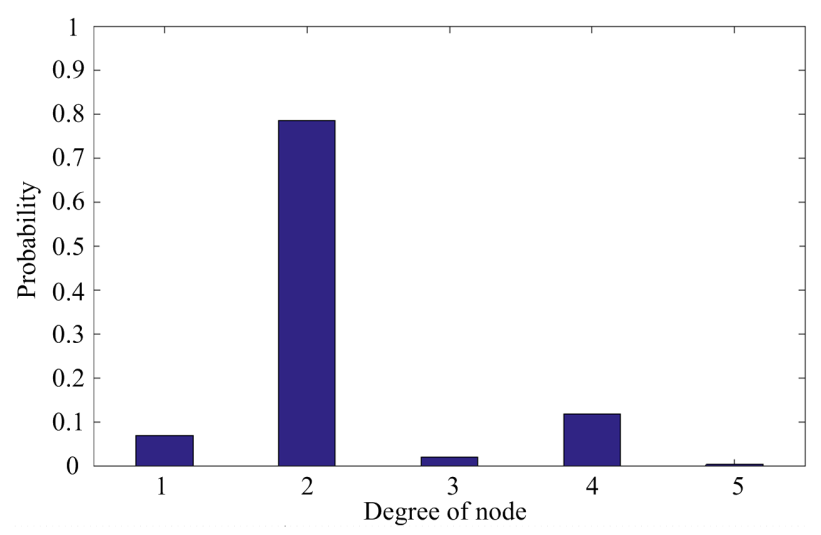

Fig. 3. (Color online) Degree distribution.

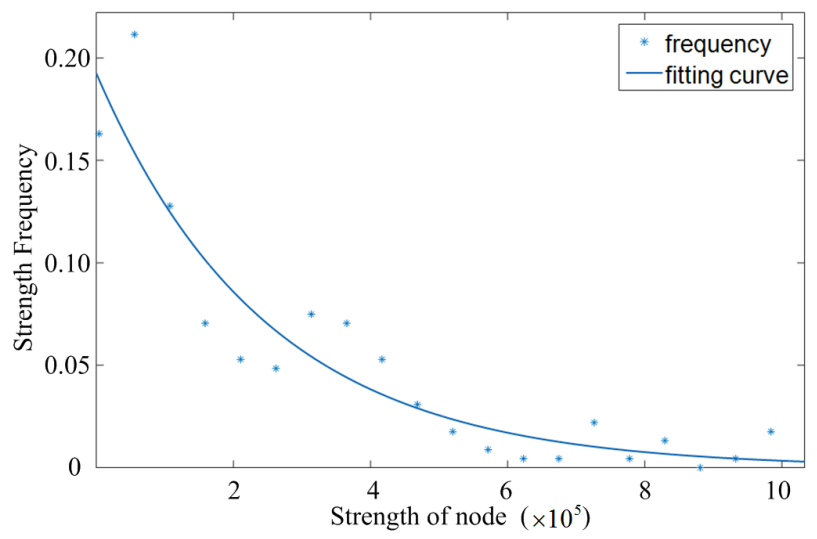

Fig. 4. (Color online) Node strength distribution.

passenger flow volume of a section of the entire network is considered as the strength of the connection between two neighboring station nodes. The calculation results suggest that three stations' node strengths exceed 900,000 passengers: Fuxingmen, Jianguomen and Guomao. $80 \%$ of node strengths in the network are less than 200,000 passengers. The distribution of node strength is shown in Fig. 4.

Because the maximum of the station is five in Beijing urban rail transit network, there are only five points in degree distribution. So the fit regression analysis on the degree distribution probability of the five nodes is no more used and only the fit regression analysis on the node strength distribution of the weighted network is carried out. The resulting fit values are shown in Eq. (11). $R^{2}$ is close to 1, so the fitting results are good.

$$
f(x)=0.193 e^{-4.038 \times 10^{-6} x}, \quad R^{2}=0.87 .
$$




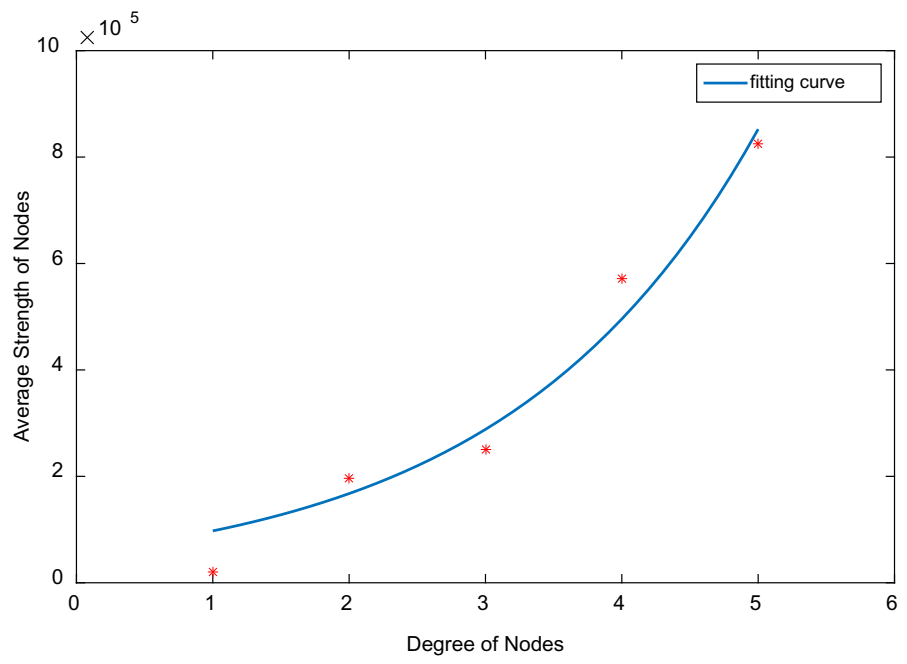

Fig. 5. (Color online) The relationship between average node strength and node degree.

Clearly, the distributions of node strength appears to show the long-tail phenomenon. This means that nodes of large node strength are few in the network, whereas nodes of small node strength are common. The significance of stations with a degree of one is enhanced because the weighted network and node strength distribution become greater for the same reason. By adding weights, the disadvantage of ignoring the differences between the degrees of stations in the unweighted network is mitigated. The average node strength and degree of nodes with the same degree are fitted, as shown in Fig. 5. Their relationship equation is given in Eq. (12), which suggests that stations of larger degree sustain greater passenger flow volume.

$$
f(x)=5.669 \times 10^{4} \times e^{0.542 x}, \quad R^{2}=0.96 .
$$

The order of stations based on values of degree and node strength are shown, respectively, in Figs. 6 and 7. The larger the degree or node strength, the larger the dot in the figures. The large red circles indicate the stations with the largest values. In the unweighted network, the degree can only be divided into five levels, and Xizhimen station has the highest level. In contrast, in the weighted network, the station at the highest level is Fuxingmen station and Xizhimen is the fifth, based on node strength. The order of Nanlishi Road station, which has a degree of two and is of low order in the unweighted network, is raised to 11th in the weighted network.

\subsubsection{Average path length}

The distributions of average path length in the unweighted and weighted networks are shown in Fig. 8. The fitting results are shown in Eqs. (13) and (14). $R^{2}$ is close 


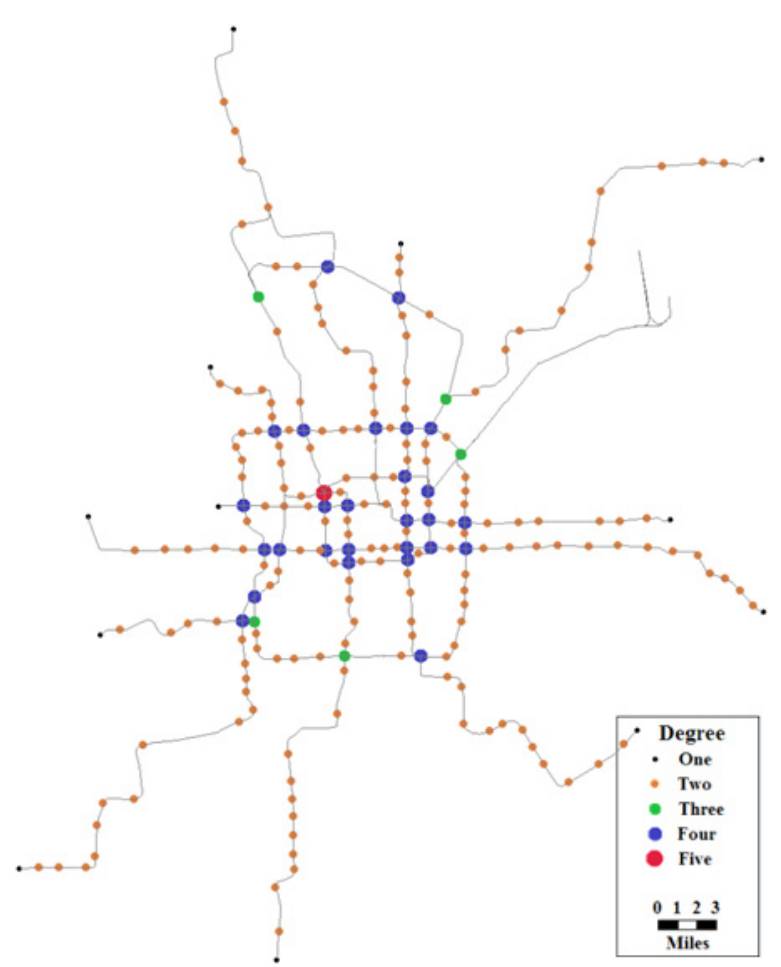

Fig. 6. (Color online) GIS display based on the order of degrees.

to 1 , so the fitting results are good.

$$
\begin{aligned}
& f(x)=0.024 e^{-\left(\frac{x-9.061}{6.736}\right)^{2}}+0.037 e^{-\left(\frac{x-16.85}{11.24}\right)^{2}}, \quad R^{2}=0.99 \\
& f(x)=0.048 e^{-\left(\frac{x-13.25}{12.72}\right)^{2}}, \quad R^{2}=0.99
\end{aligned}
$$

By comparing average path length in the unweighted and weighted networks, we find that in the unweighted network the probability of path length being 11 is largest, whereas in the weighted network the probability of path length being 13 is largest, which is relatively longer than that in the unweighted network. Although passengers are willing to choose the shortest path, when passenger flow volume increases and congestion degree increases accordingly, they may choose a longer path in order to pursue a relatively more comfortable trip. In an OD trip with path length less than eight, the probability of a short-distance trip in practice (in the weighted network) is higher than the ratio of short-distance OD pairs in the network structure (in the unweighted network). In an OD trip with path length larger than 23, actual trip probability is lower than the ratio of long-distance OD pairs. This means that the attraction of middle- or short-distance trips in urban rail transit is larger than that of long trips. 
Y. Hu et al.

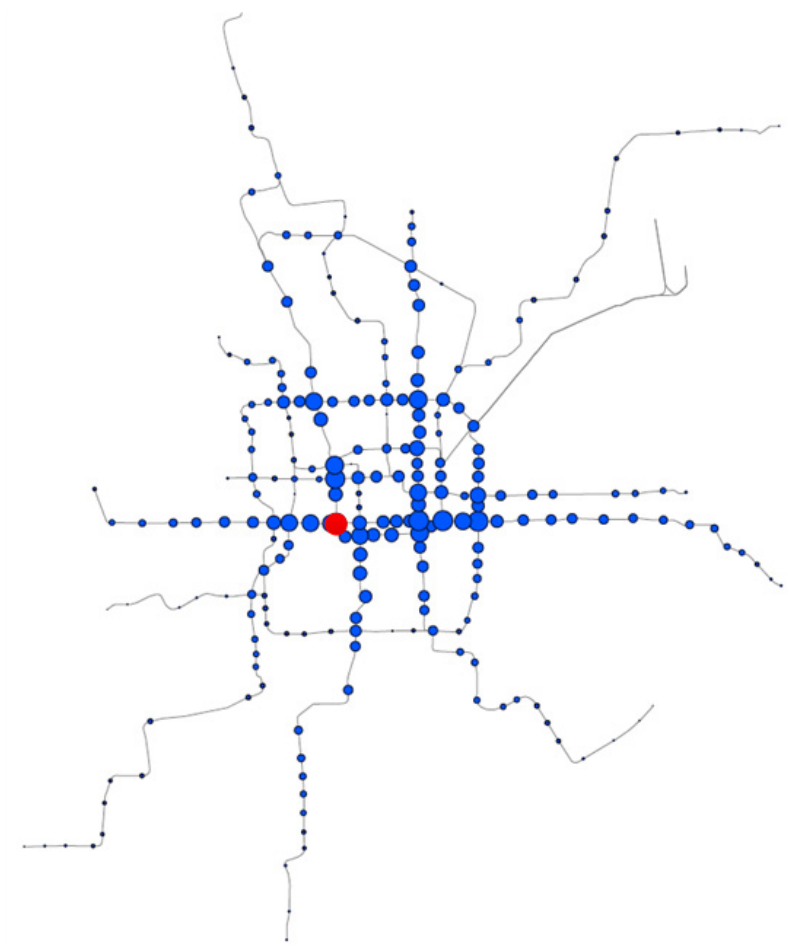

Fig. 7. (Color online) GIS display based on the order of node strength.

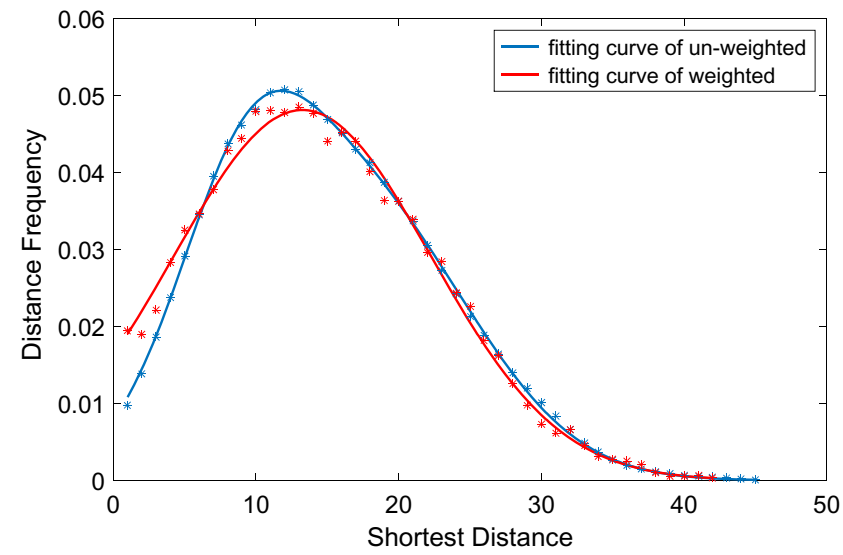

Fig. 8. (Color online) Distributions of average path length in the unweighted and weighted networks.

\subsubsection{Distribution of node betweenness}

The values of node betweenness in unweighted and weighted networks are calculated and fitted as shown in Fig. 9. The fitting results are shown in Eqs. (15) and (16). 


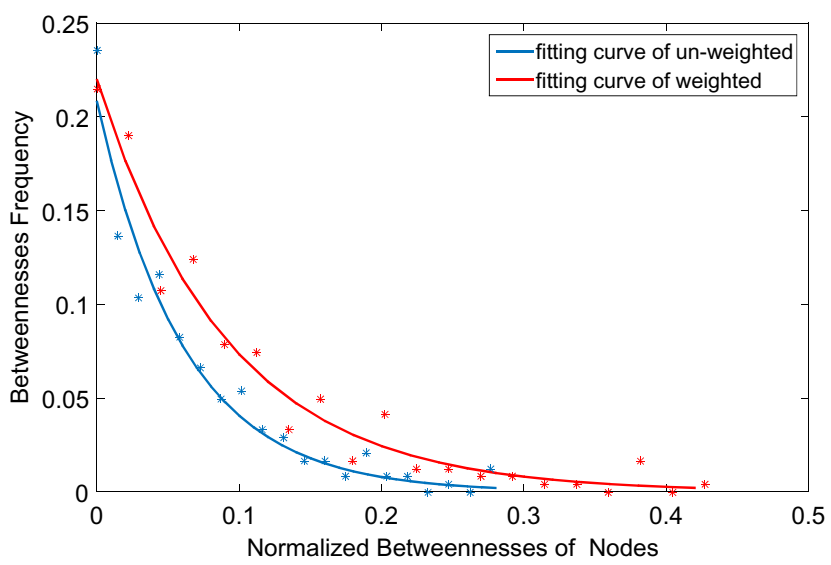

Fig. 9. (Color online) Normalized betweenness of nodes in the unweighted and weighted networks.

$R^{2}$ is close to 1 , so the fitting results are good.

$$
\begin{array}{ll}
f(x)=0.209 e^{-16.32 x}, & R^{2}=0.96 \\
f(x)=0.220 e^{-10.98 x}, & R^{2}=0.96 .
\end{array}
$$

The form of the node betweenness distribution in the unweighted network is similar to that in the weighted network. The probability of stations with large betweenness is low, whereas the probability of stations with small betweenness is high. After introducing passenger flow into the system, the betweenness of the weighted network is larger than that of the unweighted network, and the distribution of betweenness for each station node is also enlarged. Thus, the maximum betweenness of the weighted network is far larger than that of the unweighted network. Furthermore, the probability of the distribution of station nodes with the same betweenness in the weighted network is lower than that of the unweighted network.

\subsection{Comparison of robustness parameters in unweighted and weighted networks}

The Beijing Subway was simulated under continuous attack, considering three types of attack strategies: stochastic attacks, largest degree or largest strength nodebased attacks and highest betweenness node-based attacks. By analyzing robustness parameters, global efficiency and average path length, we determined the relevance and differences in these parameters between the unweighted and weighted networks. MATLAB was used for programming to calculate robustness parameters after every attack until the network was damaged throughout. The variation of robustness parameters is illustrated in Figs. 10-15. The smaller figures in Figs. 10-15 show the relevance of robustness parameters in the unweighted and weighted networks under the same attack strategy. 


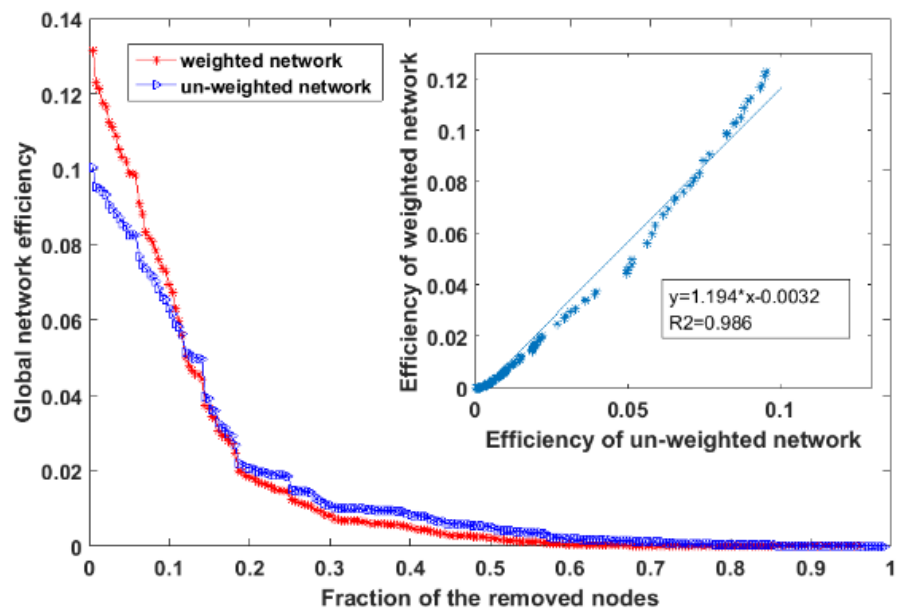

Fig. 10. (Color online) Variation of global efficiency under stochastic attack.

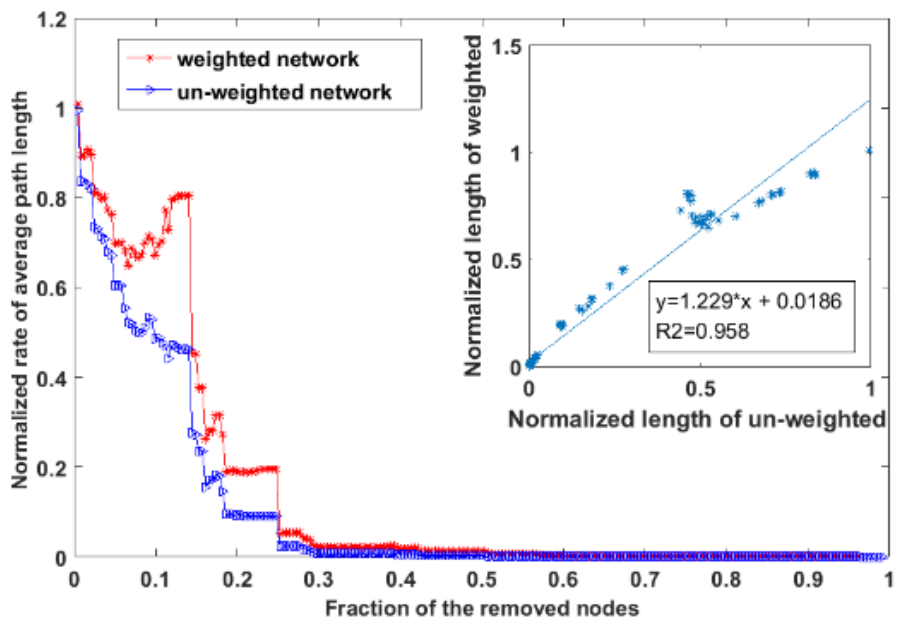

Fig. 11. (Color online) Variation of average path length under stochastic attack.

By analyzing the calculated results, we find differences from the original physical network by introducing passenger flow as the network's weight:

(1) As the figures above show, when $20 \%$ of the stations are attacked, most of the network's function has been lost. The robustness parameters of the two types of networks appear to be linearly dependent and have similar variation trends. However, in the process from the beginning of the attack to $80 \%$ of the network's function being damaged, the difference between variations of these two networks is significant. Thus, the influence of passenger flow should not be ignored. 


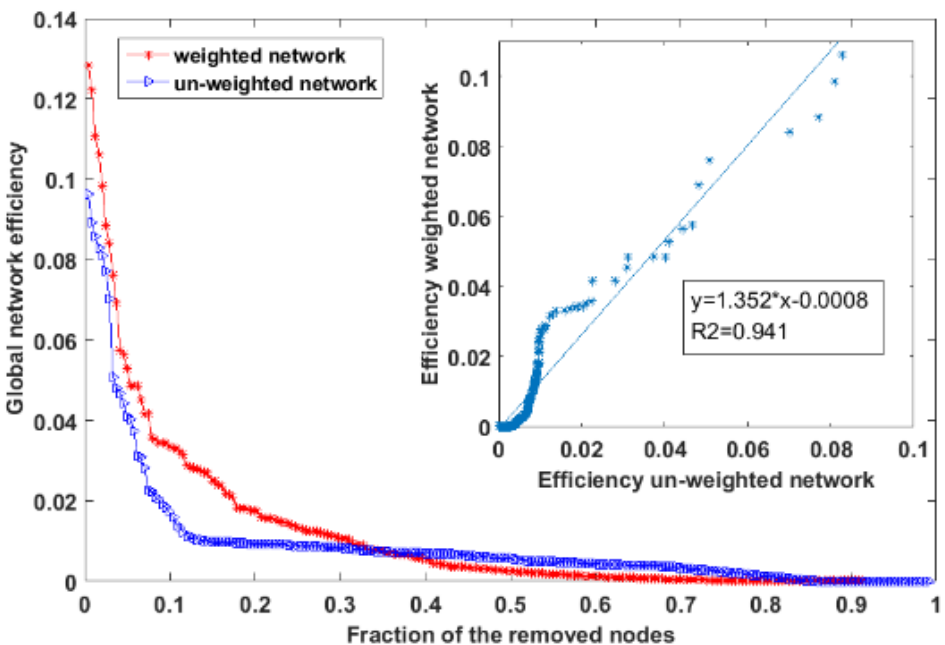

Fig. 12. (Color online) Variation of global efficiency under largest degree or largest strength node-based attack.

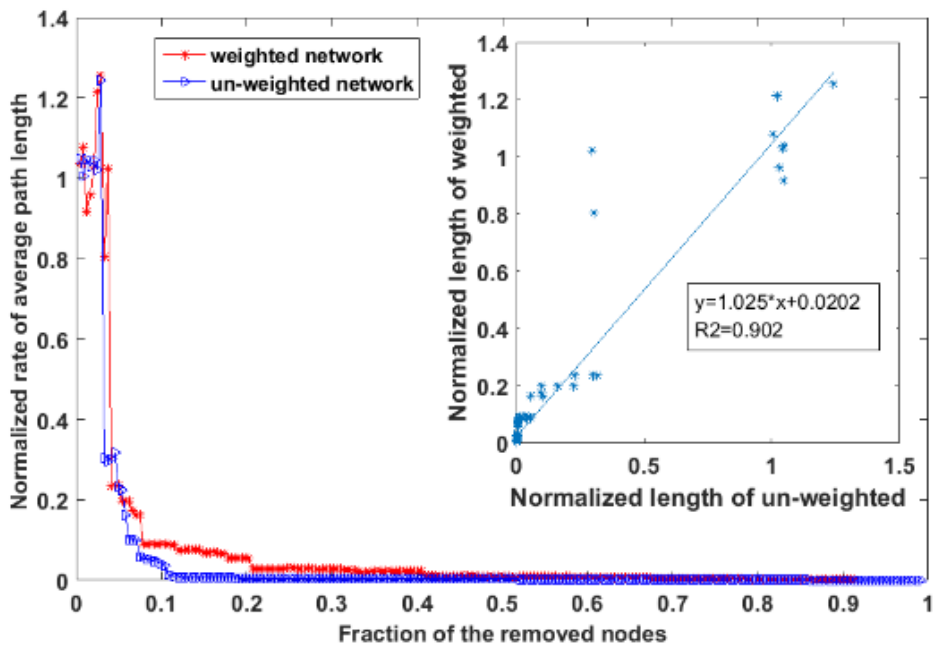

Fig. 13. (Color online) Variation of average path length under largest degree or largest strength node-based attack.

(2) The initial global efficiency of the weighted network is larger than that of the unweighted network, which means that passenger flow can enhance the connections between stations and improve global efficiency of the network. Nonetheless, owing to continuous attack, the rate of decrease of global efficiency for the weighted network is larger than that of the unweighted network. This suggests that Beijing Subway network in actual operation is weaker than the topology structure network would suggest. 


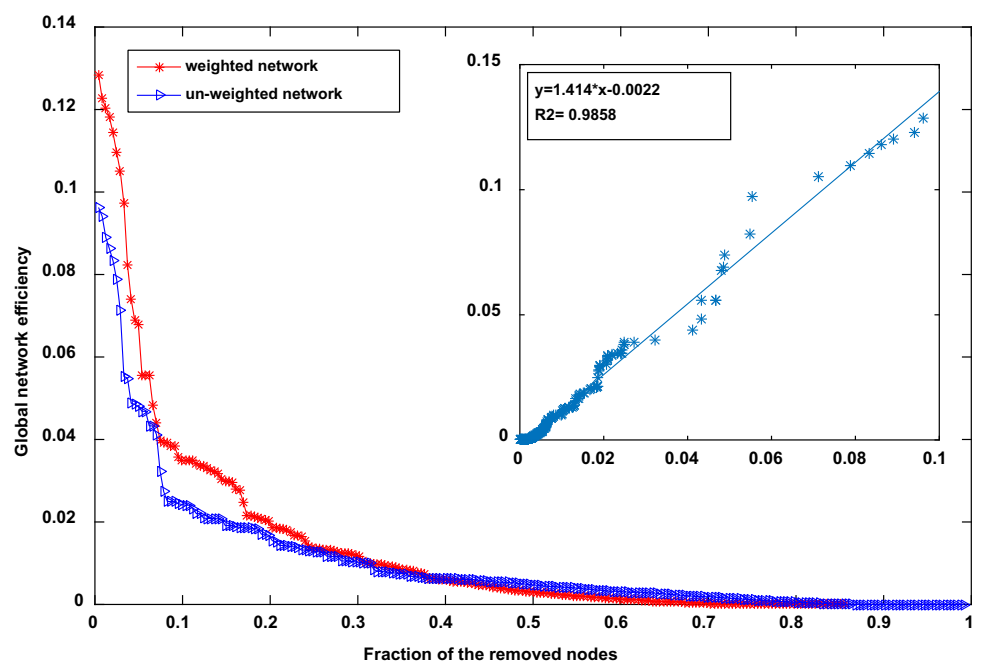

Fig. 14. (Color online) Variation of global efficiency under highest betweenness node-based attack.

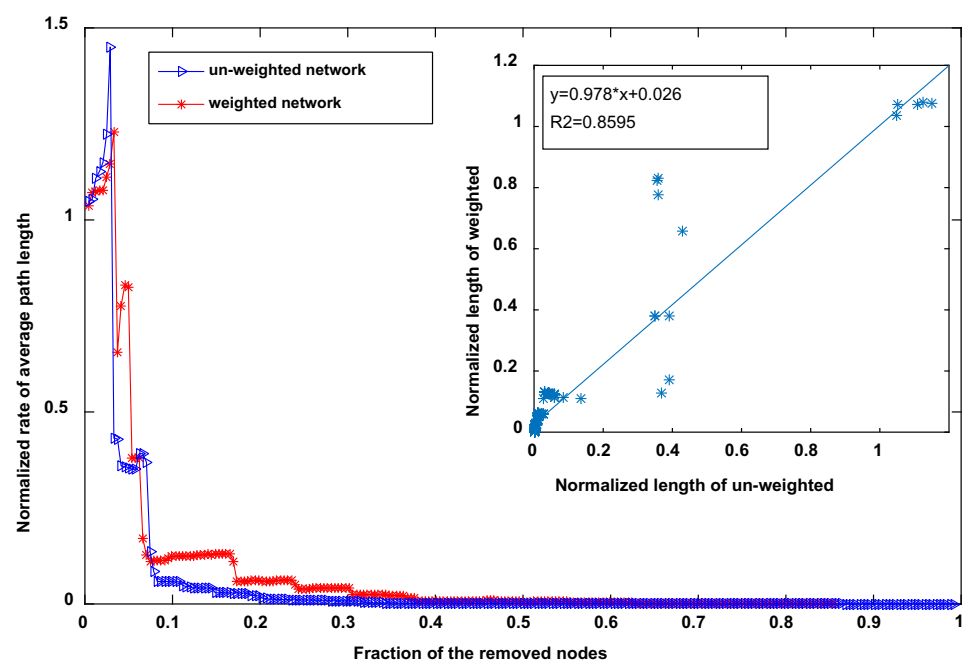

Fig. 15. (Color online) Variation of average path length under highest betweenness node-based attack.

(3) For some stations, the difference between the two types of network is remarkable. It is clear that passenger flow in a station can influence the topology characteristics of the network structure. Therefore, using an unweighted network to evaluate a networks' robustness can underestimate the importance of some stations, which may leave weaknesses open to terrorist attack. 


\section{Conclusion}

Due to the obvious difference between simple network and real rail transportation network in operation, it should be noted that the rail transportation network is built to serve passenger transportation and studying the network characteristics alone without considering the passenger flow is far away from the reality. Thus, it should be paid special attention to difference when studying the characteristics of the network with and without considering the supplement of passenger flow on the edges and nodes. In this study, the daily average passenger volume was introduced as a weight for rail transit networks. With this in mind, the essence of a weighted network was defined. Then, a complex network model for urban rail transit was developed using passenger flows as a weight. Beijing Subway was analyzed as a case study, and the complex network indicators with and without passenger volume were compared. Finally, robustness for unweighted and weighted networks were evaluated to determine their relevance and differences under three types of attack strategies. Conclusions drawn from this study are summarized as follows:

(1) The influence of passenger volume on the topology network was addressed. The indicators for evaluating the characteristics of the weighted network was established. The node degree, average path length and node betweenness distribution were analyzed. Without considering the passenger flow, the node distribution of the network fits power law distribution, while the node distribution of the network conforms to exponential distribution when passenger load is taken into account.

(2) A weighted model for analyzing complex network parameters and robustness of urban rail transit was developed. Its application to the Beijing Subway confirmed that the model is reasonable and can be generally applied. By varying the global network efficiency and average path length, the robustness of the weighted network and unweighted network was compared under the random attack and a deliberate attack to the node with the highest node degree in succession in Beijing Subway network. It is found that the global network efficiency in weighted network decreases faster than that of unweighted network. It can be concluded that the network is more vulnerable once passenger flow on it is considered. Thus the influence of passenger flow should be taken into account in further study so as to provide a more accurate network security prediction.

(3) Passenger flow weight can act as an amplification coefficient for network characteristics. We compared the robustness in terms of the initial value and variation of evaluation indicators such as global efficiency and average path length. In normal operation circumstances, passenger flow can enlarge the importance of certain stations and lines and change the global efficiency of the network. If the network is attacked, high passenger flow can aggravate the damage done on the network.

(4) A weighted network can determine the importance of stations in the subway system. Protection should be enhanced for significant stations in an 
antiterrorist system, reducing potential safety hazards according to the results.

Section loads were adopted in this research to weigh the influence of ridership on the behavior of rail lines as a complex network. It is easy to consider that different ridership indicators can be used as weights to investigate the network according to the purpose of the analysis. For example, using the predicted passenger flow during the planning stage, the robustness and importance order of stations in different urban rail transit networks can be analyzed. This can provide new insights for reasonably planning the scale of the network. Furthermore, based on the analysis of the influence of actual passenger flow on the entire network and on individual stations, operators can optimize passenger control methods such as train scheduling and temporary station closure. These topics should be addressed in the next step.

\section{Acknowledgment}

This research was supported by the National Natural Science Foundation of China (No. 51408029).

\section{References}

1. S. H. Strogatz, Nature 410, 268 (2001).

2. R. Albert, H. Jeong and A. L. Barabási, Nature 406, 387 (2000).

3. M. E. J. Newman and D. J. Watts, Phys. Lett. A 263, 341 (1999).

4. R. Albert and A. L. Barabasi, Rev. Mod. Phys. 74, 47 (2002).

5. P. Crucitti et al., Nature 406, 542 (2000).

6. P. Angeloudis and D. Fisk, Physica A 367, 553 (2006).

7. W. Li and X. Cai, Phys. Rev. E 69, 046106 (2004).

8. G. Bagler, Physica A 387, 2972 (2008).

9. B. Berche et al., Eur. Phys. J. B 71, 125 (2009).

10. H. Soh et al., Physica A 389, 5852 (2010).

11. P. Sen et al., Phys. Rev. E 67, 036106 (2003).

12. V. Latora and M. Marchiori, Physica A 314, 109 (2002).

13. Y. Wan, Urban Mass Transit 2, 33 (2009).

14. C. Han and L. Liu, in Proc. Int. Conf. Management and Service Science (MASS '09) (2009), pp. 1-4.

15. Z. Wang et al., J. Southeast Univ. Nat. Sci. Ed. 43, 895 (2013).

16. M. E. J. Newman, Phys. Rev. E 64, 016131 (2001).

17. A. Barrat et al., Proc. Natl. Acad. Sci. USA 101, 3747 (2004).

18. H. K. Liu and T. Zhou, Acta Phys. Sin. 56, 106 (2007).

19. S. Derrible and C. Kennedy, Physica A 389, 3678 (2010).

20. A. D.-L. Santos et al., Transp. Res. Part C Emerg. Technol. 20, 34 (2012).

21. J. J. Wu, Z. Y. Gao and H. J. Sun. Int. J. Mod. Phys. B 20, 2129 (2012).

22. A. Nagurney and Q. Qiang, Europhys. Lett. 79, 38005 (2007). 